\title{
Two beam toy model for dislocation contrast in ECCI
}

Elena Pascal ${ }^{1,2}$, Ben Hourahine ${ }^{1}$, Carol Trager-Cowan ${ }^{1}$ and Marc De Graef ${ }^{2}$

${ }^{1}$ Dept. of Physics, SUPA, University of Strathclyde, Glasgow, G4 0NG, UK.

2 Dept. of Materials Science and Engineering, Carnegie Mellon Univ., Pittsburgh PA 15213, USA.

Dislocation contrast in the SEM, as observed through electron channelling contrast imaging (ECCI), is commonly treated analogously to the contrast in the TEM. This perception is based on early studies on dislocations parallel with the surface where the surface relaxation is negligible. However, for threading dislocations (TD) that interact with the surface (normal or inclined), as is the case for nitride materials, $\mathbf{g} \cdot \mathbf{b}$ type invisibility criteria are no longer fully applicable to ECCI, especially in the forward geometry [1]. Dislocations locally change the lattice curvature and Bragg diffraction conditions in the crystal, affecting the form and diffracting behaviour of the electron wave function in that region. More explicitly, Howie and Whelan [2] have shown that dislocation contrast is the result of transitions between Bloch waves states, which, in turn, are caused by the change in the displacement field, $\mathbf{u}(\mathbf{r})$, or local "strain", around the dislocation. Dynamical models have been used successfully to both predict and characterise dislocations in ECCI [3]. Nevertheless, the behaviour of dislocation contrast in ECCI in particular, and defect diffraction contrast in the SEM in general, remains somewhat opaque.

In this work, we investigate the behaviour of contrast-causing strain as a means to provide insight into this problem. We call the dislocation strain component to which ECCI is sensitive ECCI-strain. We define it, generalising Tunstall's work [4], as being composed of the change of the $\mathrm{g}$ components of the displacement field, $\mathbf{u}_{g}$, in the direction of the incident beam $\mathbf{r}_{i n c}$ (change in lattice curvature) plus a small contribution (weighted by the Bragg angle $\theta_{B}$ ) of its change in the direction of the diffraction condition, g:

$$
\text { ECCI-strain }=\frac{\partial u_{g}}{\partial r_{i n c}}+\theta_{B} \frac{\partial u_{g}}{\partial r_{g}} .
$$

Negative (blue) and positive (red) isosurfaces of equal absolute value of the ECCI-strain are shown in Fig. 1 for both an edge (a) and a screw (b) normal threading dislocation in a wurtzite crystal for a $50^{\circ}$ degree tilted sample. Notice that, not only can the surface relaxation be very large compared to the bulk effect for an edge dislocation, but the sign of the strain can flip.

We can also examine whether the dislocation character or the diffraction condition will have the greater qualitative effect on the strain profile and therefore contrast. Fig. 2 shows a top sample view for an edge TD ECCI-strain isosurfaces. In the same diffraction condition, different edge dislocations (Fig. 2 (a) and (b)) will show ECCI-strain profiles rotated to each other, somewhat following the Burger vector. On the other hand, the same edge dislocation in different diffraction conditions will change less drastically its strain orientation but it will affect significantly its intensity profile ((a), (e), (f) and $(\mathrm{g}))$. As expected, flipping the direction of the diffraction vector ((c) and (d)) or the Burger's vector ((b) and (c)) flips the positive and negative strain regions.

The dislocation contrast behaviour in ECCI will then follow closely the behaviour of the strain [5]. Note that numerical predictions of contrast involve the integration along the incident beam direction of the backscattering probabilities. For inclined samples, the contrast projection will be. therefore, slightly distorted with respect to the strain profiles shown here in the sample frame.

\section{References}

[1] E Pascal et al, Mat. Today: Proceedings, 5, 2018.

[2] A Howie and M J Whelan, Proc. Royal Soc. Lond. A 263.1313, 1961. 
[3] Y N Picard et al, Ultramicroscopy 146, 2014.

[4] W J Tunstall et al, Phi. Mag. 9.97, 1964.

[5] E Pascal, PhD Thesis, University of Strathclyde, 2019.

[6] EP and MDG acknowledge an ONR Vannevar Bush Faculty Fellowship (N00014-16-1-2821), and the computational resources of the Materials Characterization Facility at CMU, grant MCF-677785.
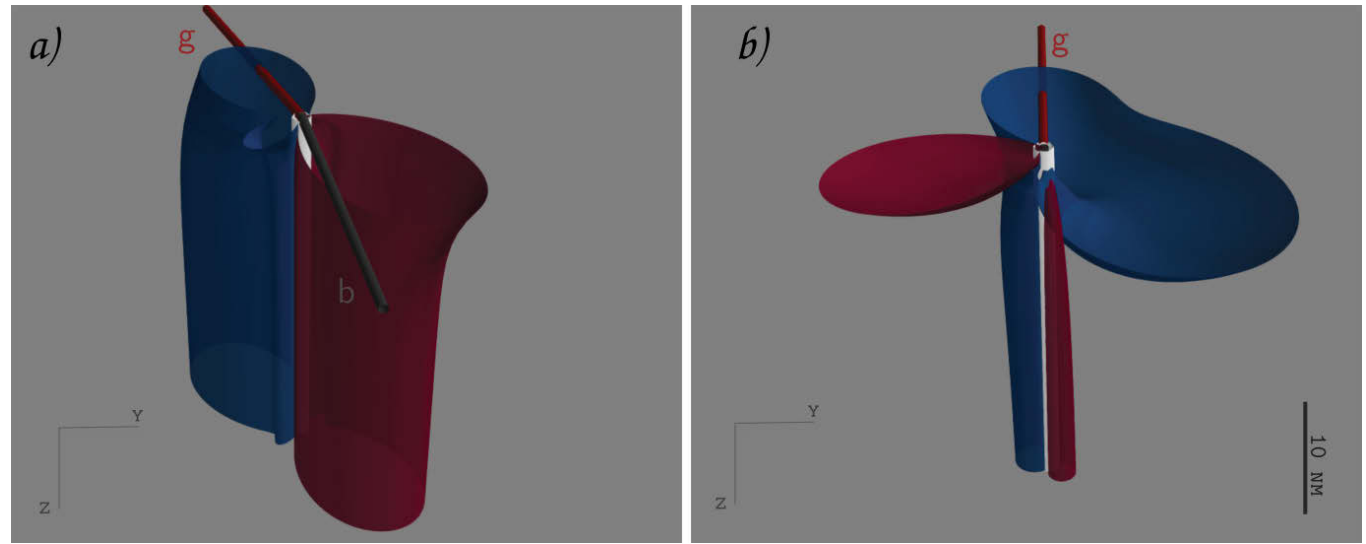

Figure 1: Side view of equidistant ECC-strain field isosurfaces for a) an Edge with Burger vector direction $\mathbf{b}$ (grey rod) and b) a Screw threading dislocation in a wurtzite crystal. The absolute values of red and blue isosurfaces is the same (equidistant). The diffraction condition is indicated by sample surface projection of $\mathbf{g}$ (red rod). (yz) indicate the sample coordinate frame where $z$ is normal to the surface and $x$ is the rotation axis.
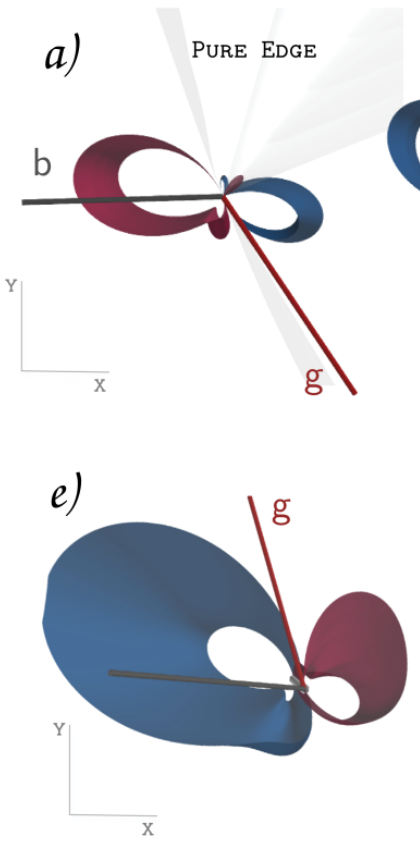

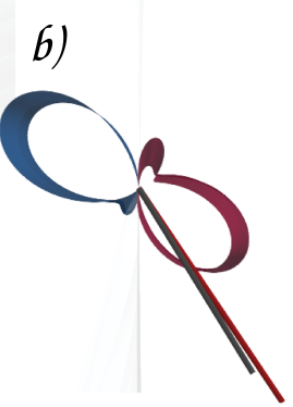

f)

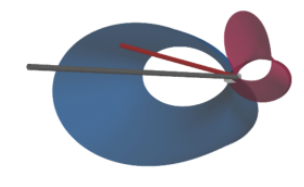

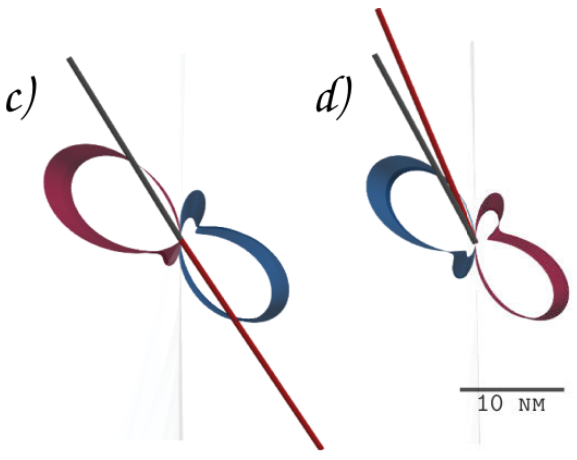

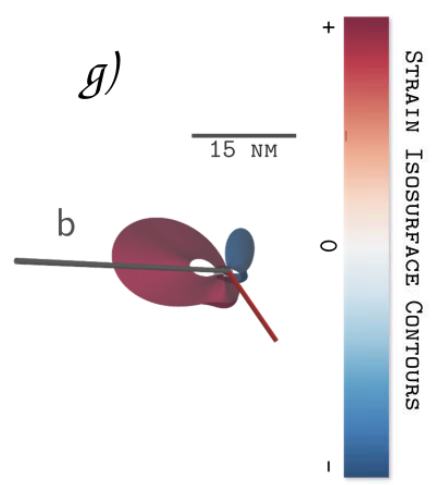

Figure 2: Top view of equidistant ECC-strain isosurfaces for a pure edge dislocation in a wurtzite crystal in different orientations indicated (see above caption). (xy) indicate the sample coordinate frame. The white curves are the zero value isosurfaces. Note that top and bottom rows have slightly different scales. 